\title{
THE TRAJECTORY ANALYSIS OF BEVEL PLANETARY GEAR TRAINS
}

CONF-910957--4

Chen-Chus Lin and Lung-Wen Tsal

DE92 040898

\author{
Mechanical Engineering Deparment \\ and \\ Systems Research Center \\ The University of Maryland \\ College Park. MD 20742
}

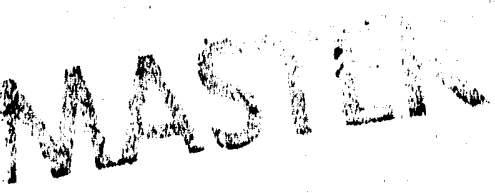

\section{ABSTRACT}

In this paper, the trajectory of bevel pianetary gear trains has been studied. The pararnetric equntious of trajectory are derived. It is shown that the trajectory generated by a tracer point on the planet of a bevel planetay gear train is analo. gous to that of a spur planetary gear train. Two cases, gear ratio equal to one and swo, are presented in detail including the geometric description, plane of symmetry, extent of trajectory, number of nodes (cusps) and their locations. The criteria for the existence of cusps are verified algeibaically, and interpreted from geometrical point of viuw.

\section{INTRODUCTION}

In the theory of Kinematics of Mechanis'ns, trajectories generated by planetary gear trains form an important family of curves, hnown as Epitrochoids or Hypotrochoids, depending on whether the gearing is external or internal (Lawrence 1972, and Hunt 1978). If the tracer point is on the pitch circle of the planet gear, the curves are called Epicy'cloids or Hypocycloids. Enowledge of planar epicyclic curves dates back to two thousand yeary ago, when ancient astronomer Hipparchos (180-125 B.C.) used epicycles to describe the paths of planets. Throughout the history of science and technology, these curves aiso found ap. plications in mechanics, optics, architecture, mechanisms, etc (Brieskiorn 19S6, Jensen 1965, Pollitt 1960, and Spragg and Tesar 1.971).

In this paper, the geometry of trajectories generated by a tracer point on the planet of bevel plavetary gear trains is stud. ied. This study serves as a precursor to the ivorispace analysis of a neiv class of gear-coupled, three DOF (Degrees Of Ftcedom), four-jointed wrist mechanisms. Before further discussion, it is heipful to review briefly the trajectories generated by a 3 p planetary gear train because of the analogy between them.

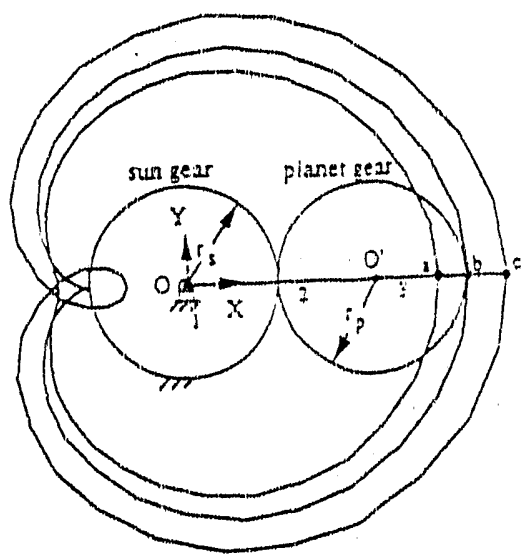

Figure 1: Limacons traced by a spur planetary gear train.

Figure 1 shows a one-DOF spur planetary geal trai: which consists of three links, two turning pairs, and one ge pair. The input link of the system is the carrier (link 2). T! sun gear is fixed to the ground (link 1), while the planet (link 3 revolves around the sun gear as the carrier rotates. Let the tad of the sun and planet gears te $r_{z}$ and $r_{p}$ respectively. Then, th length of the carrier, $l_{2}$, is equal to $r_{1}+r_{p}$. Let the distanc from the tracer point to the center of the planet gear be $l_{3}$ an let the initial position be as shown in Fig. 1 (zero phase angle Then the position of the tracer point can be described by 11 . following parametric equatious:

$$
\begin{aligned}
& \left.z=l_{2} c O_{2}+l_{3} c \mid\left(1+r_{3} / r_{p}\right) \theta_{2}\right\} \\
& \left.y=l_{2} s O_{3}+l_{3} s \mid\left(1+r_{3} / r_{p}\right) \theta_{3}\right\}
\end{aligned}
$$

where $\theta_{2}$ is the inclination angle of $O^{\prime}$ (link 2), and where denotes the cosine function and s the sine function.

This has been accepted for publication in the ASME Joumal of Mechanical Design and for presentation at the ASME 1991 Design Automation Conference, moiami 9/22-25/91 


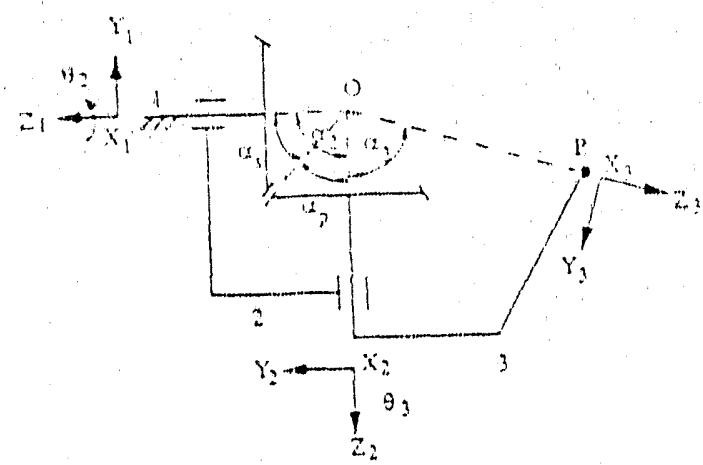

Figure 2: Bevel planctay gen unim.

Let the radii of both gears be one, and the lengths of 13 for tracer points a. b, and c, be 0.7. 1.0, and 1.t. tespectively. Then the trajectory traced by point $c$, which lies outside the pitch circle of the planet gear, has a noda. while the trajectory traced by point b. which lies on the pitch ircle of the phanet gear, has a cusp. as shown in Fig. 1. The curre is called a limacon when $r_{p} / r_{p}=1$. and is called a cardiciti when $r_{s} / r_{p}=h_{3} / r_{p}=1$. The geometric properties of linacons and carlioids can be found in (NeCarthy 1045, or Butchart 1945). Fol other ratios of $r_{s} / r_{p}$ and $l_{3} / r_{p}$, see (Lawrence,1972) for the shape of curves.

\section{PARAMETRIC EQUATIONS OF TILE TIAJECTORY}

Figure 2 shows the schernatic of a bevel planetary gear train. The trajectory generated by a tracer point on the planet geat lies on a sphere centered about point $O$, the common apex of the pitch cones formed by the sum ind planct gears.

The link coordinate systems are denned according to the D-H (Denavit.Hartenberg, 1955) convention. The origin of each coordinate system is located at the center 0 . However, they have been sketched away from the center for the reason of clarity. The $\left(X Y^{\prime} Z\right)_{1}$ coordinate system is fixed to the base link with the $X_{1}$-axis pointing out of the paper and the $Z_{1}$-axis pointing along the first joint axis. We have cliosen the stretched out, joint axes coplanar configuration of the inechanism as the relerence position, and defined all the positive $X$-axes to be pointing out of the paper. The joint angle $O_{i}$ is the angle measured from $X_{i-1}$ to $X_{i}$-axis alsout the positive $Z_{;-1}$-axis. The directions of the $Z$-axes are chosen in such a way that twist angles, $\alpha_{2}$ and $\alpha_{3}$, are less than $\pi$ and greater than zero. The twist angle $\alpha_{i}$ is the angle moasured from $Z_{i-1}$ to $Z_{i}$-axis about the positive $X_{i}$ axis. Let $\alpha$, and $\alpha_{p}$ denote the cone angles of the sun gear and planet. gear, respectively, and let $n=\sin c_{n} / \sin \alpha_{p}$ be the gear ratio, then,

$$
n=-\frac{\dot{\theta_{32}}}{\hat{O}_{12}}=\frac{\dot{\theta_{32}}}{\hat{O}_{31}}
$$

where $\dot{\theta}_{i j}$ is the magnitude of the angular velocity of link $i$ with respect to link $j$. In what foilows, we shall use $\dot{\theta}_{i}$ to denote $\dot{\theta}_{i, i-1}$ for brevity.

lntegrating Eq. (2), yields

$$
O_{1}=n O_{2}+O_{p}
$$

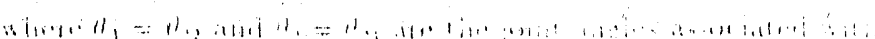

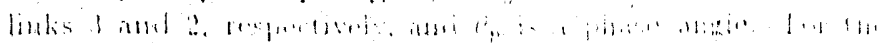

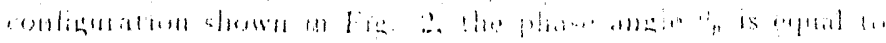
(") 111

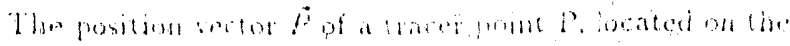

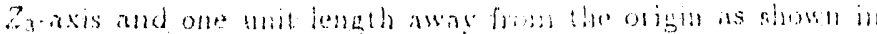

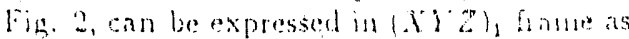

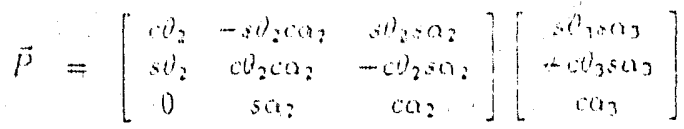

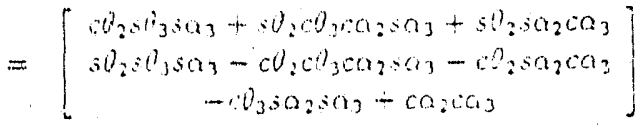

where $\theta_{3}$ is related to $\theta_{2}$ by Eq. (3). The point P will thace a curve as $\theta_{2}$ varies from $-\rightarrow$ to $\pi$

\section{EFTECT OF TIE PHASE MVGLE}

Ne shall discuss the eftect of the phine angle $\theta_{\text {, }}$ on the trajectory of $P$. Let $\theta_{p}=0$, then $\theta_{3}=n 0_{2}$, and Eq. (t) becomes

$$
\vec{P}=\left[\begin{array}{c}
c \theta_{2} s\left(n \theta_{2}\right) s \alpha_{3}+s \theta_{2} c\left(n \theta_{2}\right) c \alpha_{2} s \alpha_{3}+s \theta_{2} s \alpha_{2} c \alpha_{3} \\
s \theta_{2} s\left(n \theta_{2}\right) s \alpha_{3}-c \theta_{2} c\left(n \theta_{2}\right) c 0_{2} s \alpha_{3}-c \theta_{2} s \alpha_{2} c \alpha_{3} \\
-c\left(n \theta_{2}\right) s \alpha_{2} s \alpha_{3}+c \alpha_{2} c \alpha_{3}
\end{array}\right]
$$

Now suppose $0, p 0$, then the new position vector $\vec{p}^{\prime}$ isecumes

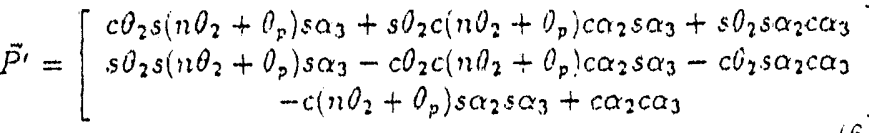

Replacing the parameter $n O_{2}$ in $\mathrm{Eq}$. (6) by $n \theta_{2}-O_{p}$ and $O_{2}$ by $\theta_{2}-\theta_{p} / n$, yield,

$$
\begin{aligned}
& \vec{p}^{\prime \prime}=\left[\begin{array}{c}
s\left(n \theta_{2}\right) c\left(\theta_{2}-\theta_{n} / n\right) s \alpha_{3}+c\left(n \theta_{2}\right) s\left(O_{2}-\theta_{p} / n\right) c \alpha_{2} s \alpha_{3} \\
s\left(n \theta_{2}\right) s\left(\theta_{2}-\theta_{p} / n\right) s \alpha_{3}-c\left(n O_{2}\right) c\left(O_{2} \ldots \theta_{p} / n\right) c \alpha_{2} s \alpha_{3} \\
-c\left(n O_{2}\right) s a_{2} s \alpha_{3}+c \alpha_{2} c a_{3}
\end{array}\right. \\
& +s\left(\theta_{2}-\theta_{p} / n\right) s \alpha_{2} c \alpha_{3} \\
& \left.-c\left(\theta_{3}-\theta_{p} / n\right) s \alpha_{2} c \alpha_{3}\right]
\end{aligned}
$$

Equation ( 7 ) can also be written as:

$$
\begin{aligned}
& \ddot{P}^{\prime \prime}=\left[\begin{array}{ccc}
c\left(O_{p} / n\right) & s\left(O_{p} / n\right) & 0 \\
-s\left(O_{p} / n\right) & c\left(O_{p} / n\right) & 0 \\
0 & 0 & 1
\end{array}\right]\left[\begin{array}{c}
c O_{2} s\left(n O_{2}\right) s \alpha_{3} \\
s O_{2} s\left(n O_{2}\right) s \alpha_{3} \\
-c\left(n O_{2}\right) s \alpha_{2} s \alpha_{3}
\end{array}\right. \\
& +s \theta_{2} c\left(n O_{2}\right) c \alpha_{2} s \alpha_{3}+s O_{2} s \alpha_{2} c \alpha_{3} \\
& -c O_{2} c\left(n \theta_{2}\right) c \alpha_{2} s \alpha_{3}-c O_{2} s \alpha_{2} c \alpha_{3} \\
& +\mathrm{Ca}_{2} \mathrm{Ca}_{3} \\
& =R\left(\cdots \theta_{p} / n, \dot{Z}_{1}\right) \ddot{P}
\end{aligned}
$$

where $R$ denotes a rotation matrix.

Equation (8) shows that adding a plyase angle has the effect of rotating the original vector and, hence, the trajectory about $Z_{1}$. axis in angle $-\theta_{p} / n$. Note that the shape of the trajectory is not changed under orthogonal transformation. 


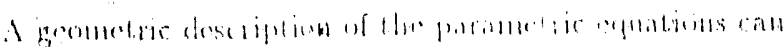

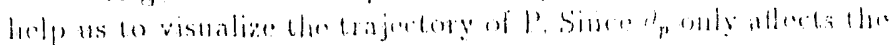

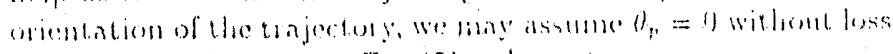
of gonemality. For $n=1$, Ey. (5) reduces 10

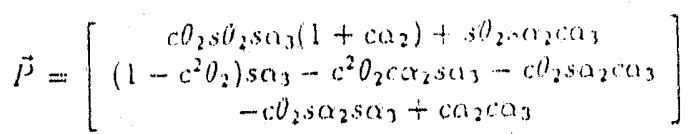

\section{Geometric Description}

Eliminating $U_{2}$ from the second and thided aquations in Eq. (9), yields

$$
\left(z+\frac{k_{2}}{2 k_{1}}\right)^{2}+\frac{k_{3}}{k_{1}}\left[y-\left(\frac{k_{3}^{2}}{k_{1} \dot{k}_{3}}-\frac{k_{4}}{k_{3}}\right)\right]=0
$$

where

$$
\begin{aligned}
& k_{1}=c \alpha_{2}+1 \\
& k_{2}=-c c \alpha_{2} c \alpha_{3}\left(1+c \alpha_{2}\right)-c \alpha_{3} s^{2} \alpha_{2} \\
& k_{3}=s^{2} \alpha_{2} s \alpha_{3} \\
& k_{1}=c^{2} \alpha_{2} c^{2} \alpha_{3}\left(1+c \alpha_{2}\right)+s^{2} \alpha_{2}\left(c \alpha_{2} c^{2} \alpha_{3}-s^{2} \alpha_{3}\right)
\end{aligned}
$$

Equation (10) represents a cylinder with a parabolic directrix in the $Y^{\prime} \cdot Z$ plare and with its elements parallel to the $X$. axis. The coefficient of $y,\left(k_{3} / k_{1}\right)$, is always positive. Therefore, the directrix parabola is convex to the positive $Y^{\prime}$ "axis direction. The :-coordinate of the apex is located at

$$
\begin{aligned}
z_{1} & =-\frac{k_{2}}{2 k_{1}} \\
& =\frac{1}{2} \cos _{3}\left(1+c a_{2}\right)
\end{aligned}
$$

The sign of $z_{\alpha}$ depends on $\alpha_{3}$. If $0<\alpha_{3}<\pi / 2$, then the apex is located above the X.Y plane: if $\pi / 2<\alpha_{3}<\pi$, then it is located below the $X-Y$ plane. The $y$-coordinate of the apex, which is also the maximum $y$-coordinate of the cylinder, is given by

$$
y_{a}=s \alpha_{3}+\frac{c^{2} \alpha_{3}\left(1-c \alpha_{2}\right)}{4 s \alpha_{3}}
$$

Surnming the squares of the $x, y, z$ components of Eq. (5), yields $x^{2}+y^{2}+z^{2}=1$. Hence, the trajectory of point $P$ is the intersection of the cylinder and a unit sphere. Figure 3 shows a typical parabolic cylinder and its intersection with a unit sphere.

\section{Extrema. Nodes, and Cusps}

For the planar motion, the geometry of epitrochoids has been described in several aspects: axes of symmetry, extent of the curve. number of node(s) or cusp(s) and their location(s), etc. Similar geometric charncteristics can also be used in the spherical motion. Since elements of the cylinder (Fig. 3) are parallel to the $Y$-axis, the intersection of the cylinder with a unit sphere centered at origin is symmetric with respect to the $Y^{\prime} \cdot Z$ plane. The extent of the trajectory can be found by soiving the extreme values of its $x, y$, and $z$ coordiuntes.

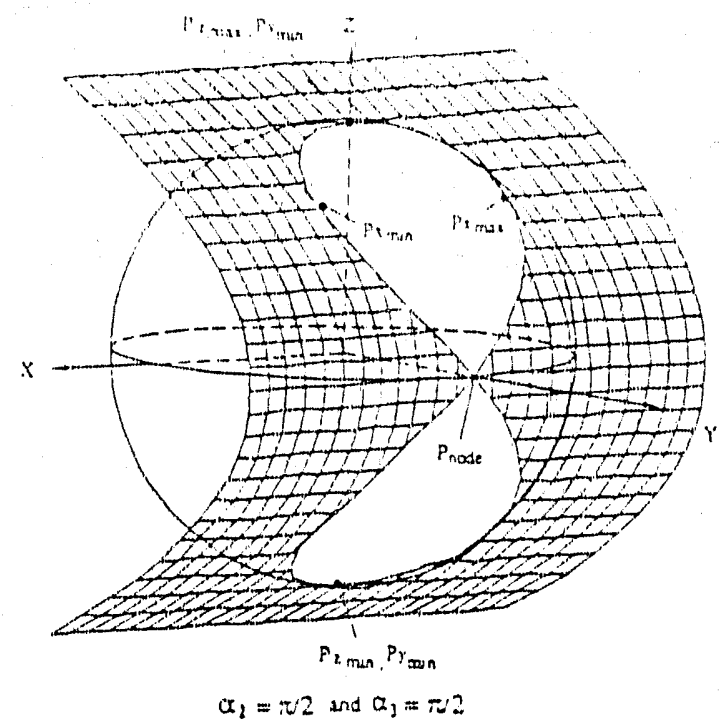

Figure 3: Intersection of a parabolic cylinder with a unit spheat

Extrema of 3. The extreme values of a can be found $b$ equating the derivalive of the $z$-component in Eq. (9) to 200 i.e.

$$
\frac{d z}{d \theta_{2}}=s \theta_{2} s \alpha_{2} s O_{3}=0
$$

From the above equation and the second-order derivative of : we conclude that at $O_{2}=\pi$ and $O_{2}=0,2$ reaches its maxi mum $z_{\text {max }}$ and minimum $z_{\text {min }}$, respectively, as shown in Eig. 3 Substituting $\theta_{2}=\pi$ into Eq. (9), yields

$$
\vec{P}_{z \max }=\left[\begin{array}{c}
0 \\
s\left(\alpha_{2}-\alpha_{3}\right) \\
c\left(\alpha_{2}-\alpha_{3}\right)
\end{array}\right]
$$

Substituting $\theta_{2}=0$ into Eq. (3), yields

$$
\vec{P}_{g_{n ! n}}=\left[\begin{array}{c}
0 \\
-s\left(\alpha_{2}+\alpha_{3}\right) \\
c\left(\alpha_{2}+\alpha_{3}\right)
\end{array}\right]
$$

Note that the two vectors with extreme values of $z$ lie on $t h$. Y't' plane, the plane of symmetry for the trajeclory. Equation: (14) and (15) imply that, the angle $\psi_{n}$, measured from $\vec{P}_{z_{\text {maz }}}$ i: $\vec{P}_{z_{m} \cdot n}$ in the clockivise direction, is given by.

$$
\psi_{z}=2\left(\pi-\alpha_{2}\right)
$$

We note that the angle $\psi_{x}$ is a function of $\alpha_{2}$ only, and $\psi_{x}=\pi$ when $\alpha_{2}=\pi / 2$.

Extrema of $x$. The extreme values of $x$ can be found by equating the first derivative of the $x$-component in Eq. (0) to zero, i.e.

$$
\frac{d x}{d \theta_{2}}=2 u_{0} c^{2} O_{2}+u_{1} c \theta_{2}-u_{0}=0
$$

where $u_{0}=s \alpha_{3}+c \alpha_{2} s \alpha_{3}$, and $u_{1}=s \alpha_{2} c \alpha_{3}$.

The solutions of Eq. (17) are

$$
\theta_{2}=\cos ^{-1}\left(\frac{-u_{1} \pm \sqrt{u_{1}^{2}+\overline{S u_{0}^{2}}}}{4 u_{0}}\right)
$$




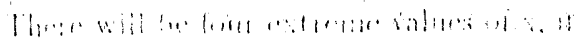

$$
-1 \leq \frac{-11 i \pm \sqrt{11+30}}{1111}-1
$$

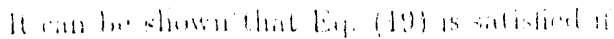

$$
\frac{12}{2} \leq a_{3} \leq \pi-\frac{112}{3}
$$

or

$$
n_{r} \leq a_{3} \leq \pi-a_{n}
$$

Exumtion (20) is the condition for he trajectory lo have four extreme values of $x$. The position of $\vec{p}$ ath extreme values of recotdinates can be obtained by substituting the solutions of $0_{2}$ from Eq. (1S) into Eq. (9). Howerer, they ane no longer in a simple form. There are two pails of $\vec{P}_{r_{n+1}}$ and $\vec{P}_{f_{m i n}}$ when Eq. (20) is satisfied, and $x_{\min }=-x_{\text {nar }}$ for each pail of extreme positions. The angle, $\psi=$, between $\vec{P}_{x_{n u x}}$ and $\vec{P}_{z_{n}, n}$ is given by

$$
1==\sin ^{-1}\left(x_{\max }\right)
$$

Points of intersection al the $) \cdot 2$ plane. Egtating the $x$ component in Eq. (O) to zero, we obtain the points where the trajectory intersects the Y.Z plane:

$$
s \theta_{2}\left[c O_{2} s a_{3}\left(1+c a_{2}\right)+s a_{2} c a_{3}\right\}=0
$$

Hence,

$$
\theta_{2}=0 \text { or } \pi
$$

or

$$
\theta_{3}=\cos ^{-1}\left(-\frac{s a_{2} c \alpha_{3}}{s \alpha_{3}\left(1+c \alpha_{2}\right)}\right)
$$

Equation (22.a) implies that the trajectory intersects the Y.Z plane at least bivice at the locations where the extreme values of 2 occur. Equation (22.b) yields two real solutions if

$$
-1 \leq-\frac{s \alpha_{2} c \alpha_{3}}{s \alpha_{3}\left(1+c \alpha_{2}\right)} \leq 1
$$

If can be shown that Eq. (23) is satisfied when

$$
\frac{\alpha_{2}}{2} \leq \alpha_{3} \leq \pi-\frac{\alpha_{2}}{2}
$$

We note that Eq. (24) is identical to Eq. (20), which means that whenever there exist four extreme values of $x$, the trajectory intersects the plane of symmetry four times. When Eq. (2t) is satisfied, iwo additional points of intersection can be found by substituting $\cos \theta_{2}$ from Eq. (22.b) into Eq. (9). Since the $y$ and $z$ components of $\vec{P}$ depend only on $\cos \mathrm{O}_{2}$, we conclude that the two additional points of intersection is a double point. This means the trajectory intersects itself and the node lies on the plane of symmetry as shown in Fig. 3. The coordinates of the node are

$$
\vec{P}_{\text {rode }}=\left[\begin{array}{c}
0 \\
s \alpha_{3} \\
c \alpha_{3}
\end{array}\right]
$$

which is function of $\alpha_{3}$ only.

Inequality (20) or (24) implies that whenever the tivist angle $\alpha_{3}$ is greater than the cone angle of the planet gear $\left(\alpha_{p}=\right.$ $\alpha_{1}=\alpha_{2} / 2$ ) and less than its suppleirient, the locus of point $p$

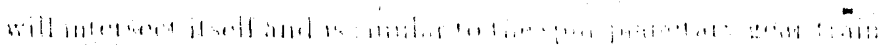
4hem

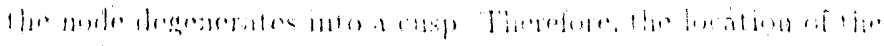

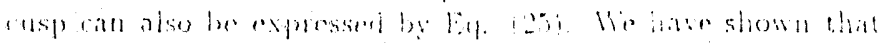

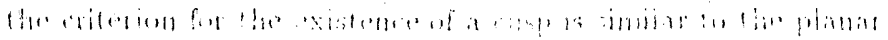

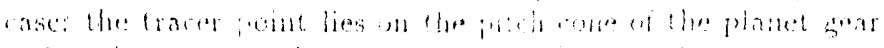
or its minor maste about the origin

Extrema of y. The exteme values of y can be comm by equating the thest leavative of the y-component in Ey. (9) 10 acro, i.e.

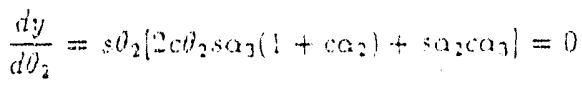

Mence.

$$
v_{2}=0 \text { or }-
$$

$O I^{\prime}$

$$
\theta_{2}=\cos ^{-1}\left(-\frac{\operatorname{son}_{2} \alpha_{3}}{2 \operatorname{son}_{3} !+\operatorname{con}_{2} !}\right)
$$

Equation (27.a) implies bhat tro exureme values of $y$ occur at the same locations where the extreme ralues of zoccur, and libey are given by Eqs. (14) and (15), respertively. If the it s deriven from Eq. (27.b) are real. then two niditional points for extreme values of $y$ exist. It can be shown that the $y$ and z-coordinates of these two extreme points are given by Eqs. (12) and (11). respectively. Since the $y$ and $z$ components of $\ddot{P}$ in Eq. 19 ) depend only on $\cos \theta_{2}$, the two extreme points have the same $y$ and : coordinates, but diftereal values of $x$.

Typical Trajectories. Figure 4 shows some trajectories generated from a few typical bevel plametary gear trains. The shape of trajectory depends on the twist angles, $\alpha_{2}$ and $a_{3}$. Gen. erally, $\alpha_{2}$ defines the maximum range in the latiludinal direction of trajectory, while $\alpha_{3}$ defines the position of the node (or cusp). When $\alpha_{2}=\pi / 2$, the angle $\psi$ : between $\ddot{P}_{2 \max }$ and $\vec{P}_{z_{\min }}$ is equal to $\pi ;$ when $\alpha_{3}=\pi / 2$, the trajectory is symmetric about the $X-Y$ plane. The trajectory has a figure-S shape if both sides of inequality in Eq. (2t) are satisfied as sjown in Figs. $4(\mathrm{a}) \cdot(\mathrm{d})$. They are analogous to planar curves generated by a spur planetary gear train (Fig. 1), which are called limacons. A limacon is defined as the curve which has the form of $r=a-b \sin \theta$ or $r=a-b \cos \theta$, where $r$ and $\theta$ are the parameters in polar coordinate system, and $a$ and $b$ are two constants. In the spherical motion, the third equation in Eq. (9) resembles the above reln. tion. We shall name the trajectorjes craced by a tracer point on a bevel planetary gear train as spherical limacons. In summary, there are three types of spherical limacons: (i) when the twist angles do not satisiy Eq. (24), the trajectories become simply. connected curves; (ii) when one of the equality-sign in Eq. (24) is satisfied, the trajectory has a cusp; (iii) when the inequality in Eq. (24) is satisfied, the trajectory has a node. Figures $4(e)$ and $(\mathrm{g})$ show two critical cases where the trajectories change to spherical cardioids, while Fig. $4(f)$ shows an exarnple for which the left-hand-side of Eq. (24) is not satisfied. 

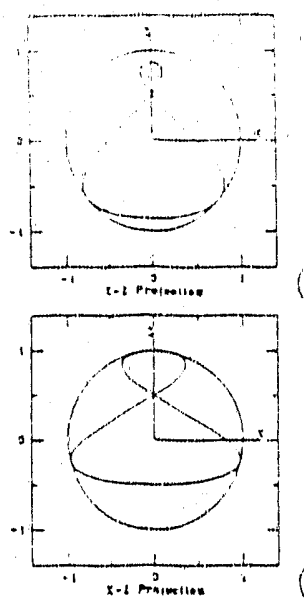

(a)

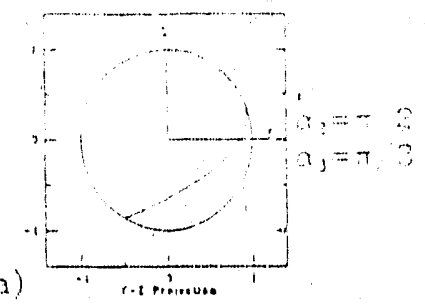

(b)
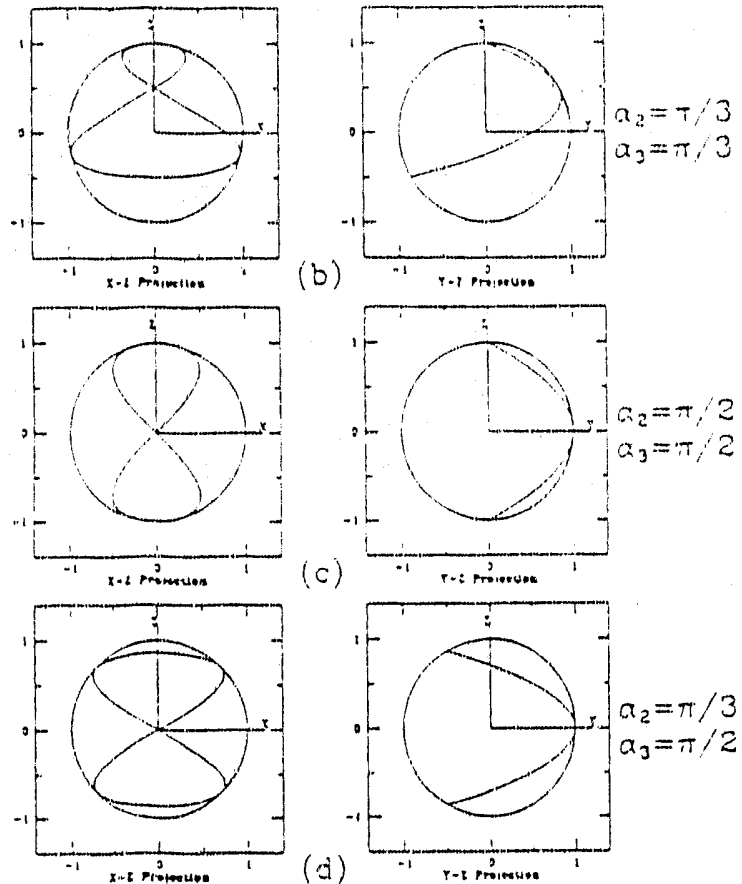

(c)

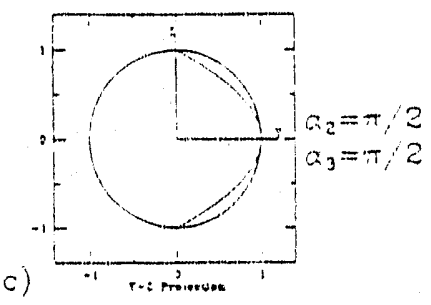

(d)
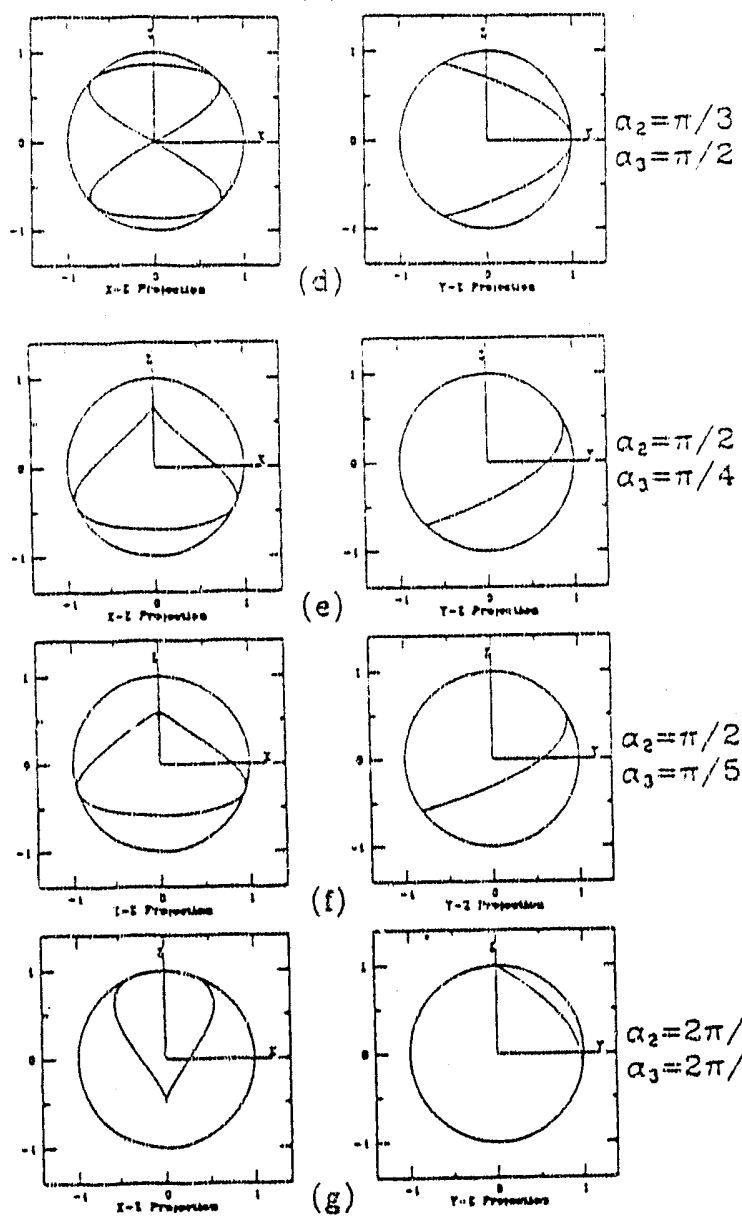

(f)

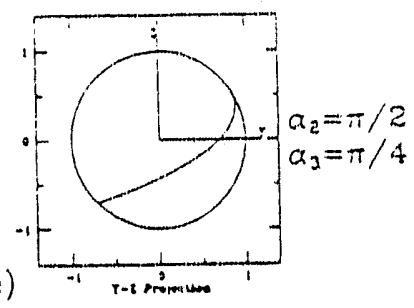

e)
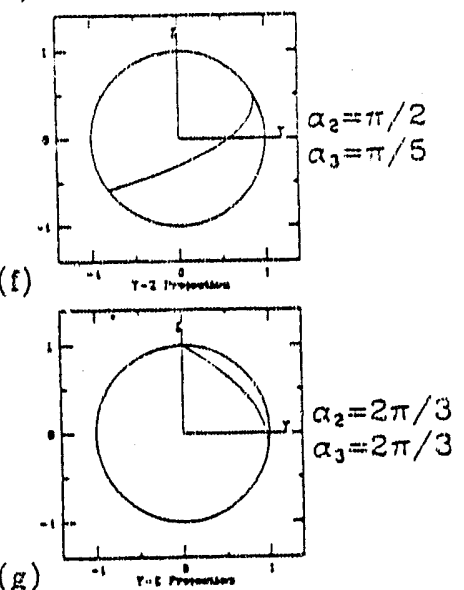

Figure 4: Spherical limarint:; $N=1$.

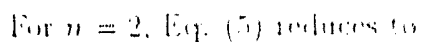

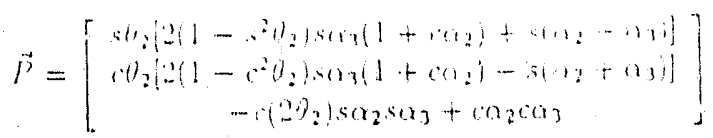

Note that $\cos \left(2 \theta_{2}\right)=2 \cos ^{2} \theta_{2}-1=1-2 \sin ^{2} \theta_{2}$.

\section{Geometric Description}

Eliminating $\mathrm{O}_{2}$ [r m the second and third equations in Eq. (2s), rieids

$$
y^{2}=\frac{1+\zeta}{2}\left|s \alpha_{3}-s \alpha_{2} c \alpha_{3}-s \alpha_{3}\left(1+c \alpha_{2}\right) \zeta\right|^{2}
$$

and eliminating $\mathrm{O}_{2}$ from the first and third equations, $\because$ ields

$$
x^{2}=\frac{1-\zeta}{2}\left\{s \alpha_{3}+s \alpha_{2} c \alpha_{3}+s \alpha_{3}\left(1+c \alpha_{2}\right){ }^{2}\right\}^{2}
$$

where $\zeta=\left(c c \alpha_{2} c \alpha_{3}-z\right) / s \alpha_{2} s \alpha_{3}$

Equations (29) represents a cylinder with a cubic directrix in the $Y . Z$ plane. Similarly, E.q. (30) aiso represents a cylinder with a cubic directrix in the $X-Z$ plane. The cubic curve is called Tschirnhausen's Cubic or l'Fospital's Cudic. Hence, we may' consider the trajectory as the intersection of a cubic cylinder and a unit sphere. From the two equations, it can be concluded that the curve has two planes of symmetry, the X'Z plane and the $Y-Z$ plane.

\section{Extrema, Nodes, and Cusps}

Extrema of $z$. When the gear ratio is equal to two, the stationary condition for $z$ coordinate is

$$
\frac{d z}{d O_{3}}=2 s\left(2 \theta_{2}\right) s \alpha_{2} s \alpha_{3}=0
$$

Considering the second-order derivative of $z$, we conclude that, when $\theta_{2}=-\pi / 2$ and $\theta_{2}=\pi / 2,2$ reaches $i$ is maximum, $z_{\text {max }}$. Substituting them into Eq. (2S), we obtain, for $O_{2}=-\pi / 2$,

$$
\vec{v}_{z \max }=\left[\begin{array}{c}
-s\left(\alpha_{2}-\alpha_{3}\right) \\
0 \\
c\left(\alpha_{2}-\alpha_{3}\right)
\end{array}\right]
$$

and for $\theta_{3}=\pi / 2$,

$$
\vec{P}_{\mathrm{zmex}}=\left[\begin{array}{c}
s\left(\alpha_{2}-\alpha_{3}\right) \\
0 \\
c\left(\alpha_{2}-\alpha_{3}\right)
\end{array}\right]
$$

Substituting $\theta_{2}=0$ into Eq. (2S), we obtain the minimum of $z$

$$
\vec{P}_{2 m i n}=\left[\begin{array}{c}
0 \\
-s\left(\alpha_{2}+\alpha_{3}\right) \\
c\left(\alpha_{2}+\alpha_{3}\right)
\end{array}\right]
$$

and for $\theta_{2}=\pi$

$$
\vec{P}_{z_{\min }}=\left[\begin{array}{c}
0 \\
s\left(\alpha_{2}+\alpha_{3}\right) \\
c\left(\alpha_{2}+\alpha_{3}\right)
\end{array}\right]
$$


Q

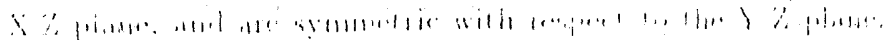

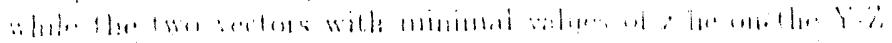

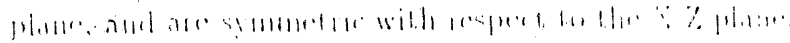

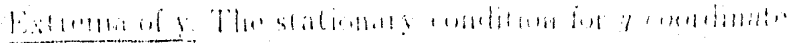
is fomat on Trefing

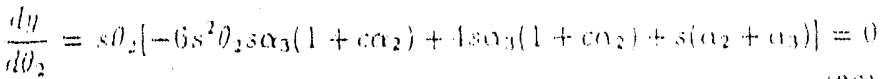

The vertors with extreme values of $y$ can tor found by substi. unting the solutions of Eq. 36) into (2S). There are at linast. two and at mosi six solutions to Eil. (Bo). When $\theta_{2}=0$ or $v_{2}=\pi$ ). the curve reaches its minimal (or maximal) value of $y$. Four additiona! solutions exist only if

$$
0 \leq \frac{2}{3}+\frac{s\left(a_{2}+a_{3}\right)}{6 \sin _{3}\left(1+c a_{2}\right)} \leq 1
$$

Equation (3T) can be simplified as

$$
-2 \leq \frac{s\left(\alpha_{2}+a_{3}\right)}{2 s \alpha_{2}\left(1+c a_{2}\right)} \leq 1
$$

Points of Intersection at $X-Z$ Plalle. The points of intersection at the X.Z plane can be obtained by equating the $y$ component of Eq. (2S) to zero, i.e.,

$$
\mathrm{CO}_{2}\left\{2 s^{2} O_{2} s u_{3}\left(1+c \alpha_{2}\right)-s\left(\alpha_{2}+\alpha_{3}\right)\right\}=0
$$

Hence.

$$
0_{2}=-\pi / 2 \text { or } \pi / 2
$$

or

$$
\theta_{2}=\sin ^{-1}\left[ \pm\left(\frac{s\left(\alpha_{2}+\alpha_{3}\right)}{2 s \alpha_{3}\left(1+c \alpha_{2}\right)}\right)^{\frac{1}{2}}\right]
$$

Equation (39.a) impies that the trajectory intersects the $X \cdot z$ plante at least twice at the locations where the maximal values of $z$ orcur. Equation (39.b) yields four real solutions if

$$
0 \leq \frac{s\left(\alpha_{2}+\alpha_{3}\right)}{2 s \alpha_{3}\left(1+c \alpha_{2}\right)} \leq 1
$$

Since the denorninator in Eq. (40) is always positive, the lelthand-side of Eq. (40) is satisfied when

$$
\alpha_{3} \leq \pi \cdots \alpha_{2}
$$

Substituting the relationship $\alpha_{2}=\alpha_{1}+\alpha_{0}$ into the right-handside of Eq. (40) and after simplification, we obtain

$$
\frac{s\left(\alpha_{3}+\alpha_{p}-\alpha_{3}\right)}{s \alpha_{3}} \leq 2
$$

Compacing it with

$$
\frac{\sin \alpha_{s}}{\sin \alpha_{p}}=2
$$

we conclude that the right, equality sign of $\mathrm{Eq}_{\mathrm{q}}(40)$ is satisfied when $\alpha_{3}=\alpha_{p}$. For a given $\alpha_{2}$, the fum tion in $\mathrm{Eq} .(40)$ is a monotonically decreasing function of $\alpha_{3}$ within the interval of $0<\alpha_{3}<\pi$. Hence, it follows that

$$
\alpha_{3} \geq \alpha_{p}
$$

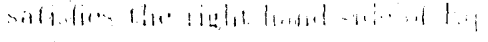

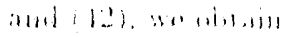

$$
11, \because 11-7 \cdots
$$

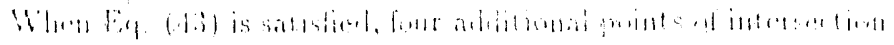

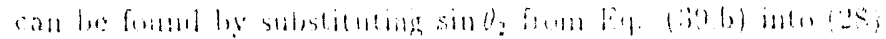

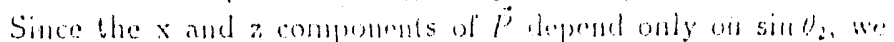
conclude that the four aditional points of intersection are two double points (nodest. When the repus sinn in Eq. (13) holds. the cwo nodes hecome two ansps.

Since Eq. (10) is a subset of Eq. (38), we can couclute that Eq. (43) atso satisties Eq. (38). Thus, when the trajectory intersects the $X-Z$ phane six times, it has six extreme values of $y$.

Points of Intersection at 12 . Plane. The points of intersection at the $Y \cdot z$ plane can be obtained by equinting the $x$ component of $E_{q}$. (23) to zero, i.e..

$$
s o_{2}\left[2 c^{2} O_{2} s \alpha_{3}\left(1+c \alpha_{2}\right)+s\left(\alpha_{2}-\alpha_{3}\right)\right]=0
$$

Hence,

or

$$
\theta_{2}=0 \text { or }-
$$

$$
0_{2}=\cos ^{-1}\left[ \pm\left(\frac{\left.s i \alpha_{3}-\alpha_{2}\right)}{2 s \alpha_{3}\left(1+c \alpha_{2}\right)}\right)^{\frac{1}{2}}\right]
$$

Equation (4ta) implies that the trajectory intersects the $X \cdot Z$ plane at least twice at the locations where the minimal values of $z$ occur. Equation $(4 ., b)$ yields four real solutions if

$$
0 \leq \frac{s\left(\alpha_{3}-\alpha_{2}\right)}{2 s \alpha_{3}\left(1+c \alpha_{3}\right)} \leq 1
$$

The right hand-side of Eq. (45) can be simplified as

$$
-\frac{s\left(c_{3}+\alpha_{p}+\alpha_{3}\right)}{s \alpha_{3}} \leq 2
$$

Comparing it with

$$
\frac{\sin \alpha_{1}}{\sin \alpha_{p}}=2,
$$

we conclude that the right equality sign of Eq. (15) is satisficd when $\alpha_{3}=\pi-\alpha_{p}$. For a given $\alpha_{2}$, the function in $E q .(45)$ is a monotonically increasing function of $\alpha_{3}$ within the intervai of $0<\alpha_{3}<\pi$. Hence, it lollows that

$$
\alpha_{3} \leq \pi-\alpha_{p}
$$

satisfies the right-hand-side of $\mathrm{Eq}$. (45). Hence, we can conclude that Eq. (45) will be satisfied if and only if

$$
\alpha_{2} \leq \alpha_{3} \leq \pi-\alpha_{p}
$$

Since the $y$ and $z$ components of $\vec{P}$ depend only on $\cos \theta_{2}$, we conclude that the four adcitional points of intersection are two double points (nodes).

Typical Trajectories. Figure 5 shows some trajectories generated by bevel planetary gear trains having geat ratio equal to two. The curves are symmetric with respect to the $X . Z$ and 

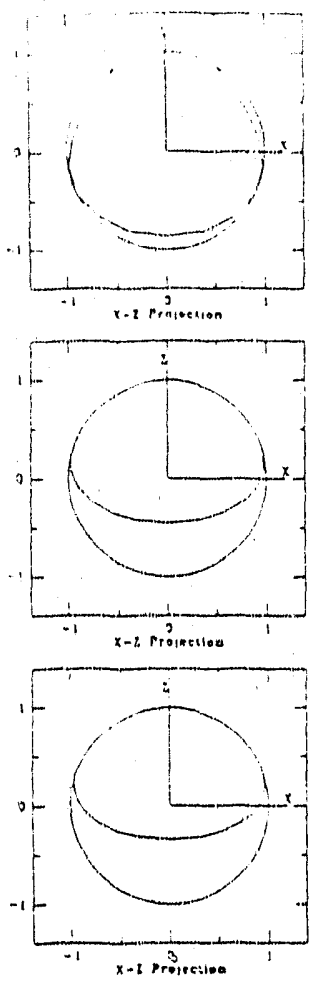

(b)

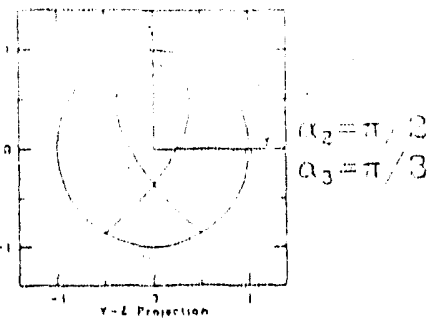

(a)

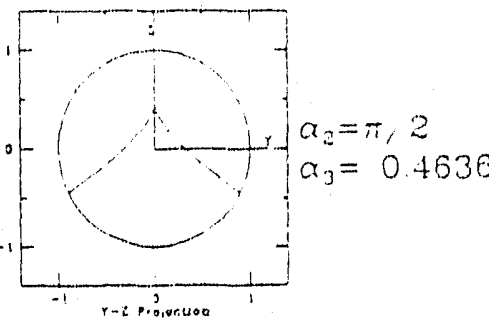

c)

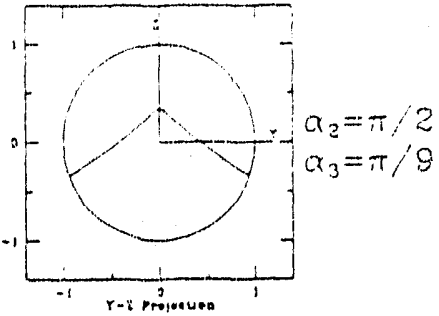

Figure 5: Typical spherical limacons, $N=2$.

$Y^{\prime}-Z$ planes. It should be noted that, given an $\alpha_{2}$, the cone angle of the planet gear is given by

$$
\alpha_{p}=\tan ^{-1}\left(\frac{s \alpha_{2}}{c \alpha_{2}+2}\right)
$$

Except for Fig. 5(c), all the other figures satisfy Eqs. (43) and/or (40). In Fig. $5(b), \alpha_{3}=\alpha_{p}$, the curve has two cusps; and in Fig. $5(e), \alpha_{3}=\pi-\alpha_{p}$, the curve also has two cusps. These curves are called spherical nephroids. Note that when Eq. (43) is satisfied, there will be a node or cusp on the Y.Z projection; Similarly, when Eq. (46) is satisfied, there will be a node or cusp on the $X-Z$ projection. Table 1 shows the number of nodes and cusps of the trajectories shown in Fig. 5 , and their relationship with Eqs. (43) and (40). It is concluded that, Eq. (43) or (46) should be satisfied for the existence of any nodes or cusps.

\begin{tabular}{|c|c|c|c|c|}
\hline & Nodex & Cusps & $a_{p}<a_{3}<\pi-a_{2}$ & $\alpha_{2}<\alpha_{3}<\pi-\alpha_{2}$ \\
\hline 7 if. $5(\mathrm{~A})$ & 2 & $a$ & $\gamma^{\prime}$ & \\
\hline$F=5(b)$ & 0 & 7 & $a_{3}=a_{p}$ & \\
\hline$F_{i s}, 5(\mathrm{Cl})$ & 0 & 0 & & \\
\hline Fir. ${ }^{3}(d)$ & 2 & 0 & $a_{3}=\pi-a_{3}$ & $a_{3}=a_{2}$ \\
\hline Fig. $=0$ & 0 & 3 & & $a_{3} \equiv \pi-a_{n}$ \\
\hline Fis.s(n) & 4 & $\overline{0}$ & $x$ & $\gamma$ \\
\hline 618.513$)$ & 3 & 0 & $a_{3}=\pi-a_{3}$ & \\
\hline $718 \mathrm{s(h)}$ & $\frac{2}{2}$ & 0 & & 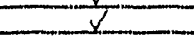 \\
\hline
\end{tabular}

Table 1: The number of nodes or cusps and their relationship with Eqs. (43) and (46).

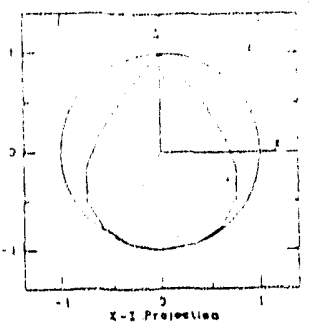

(d)
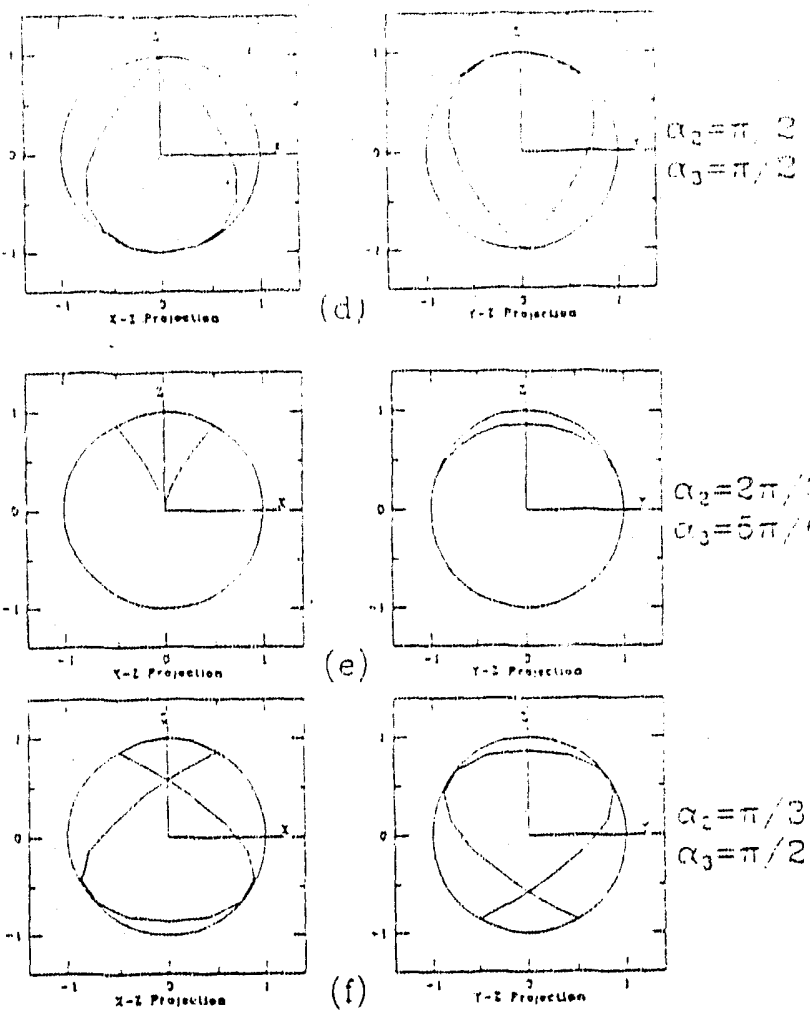

(e)
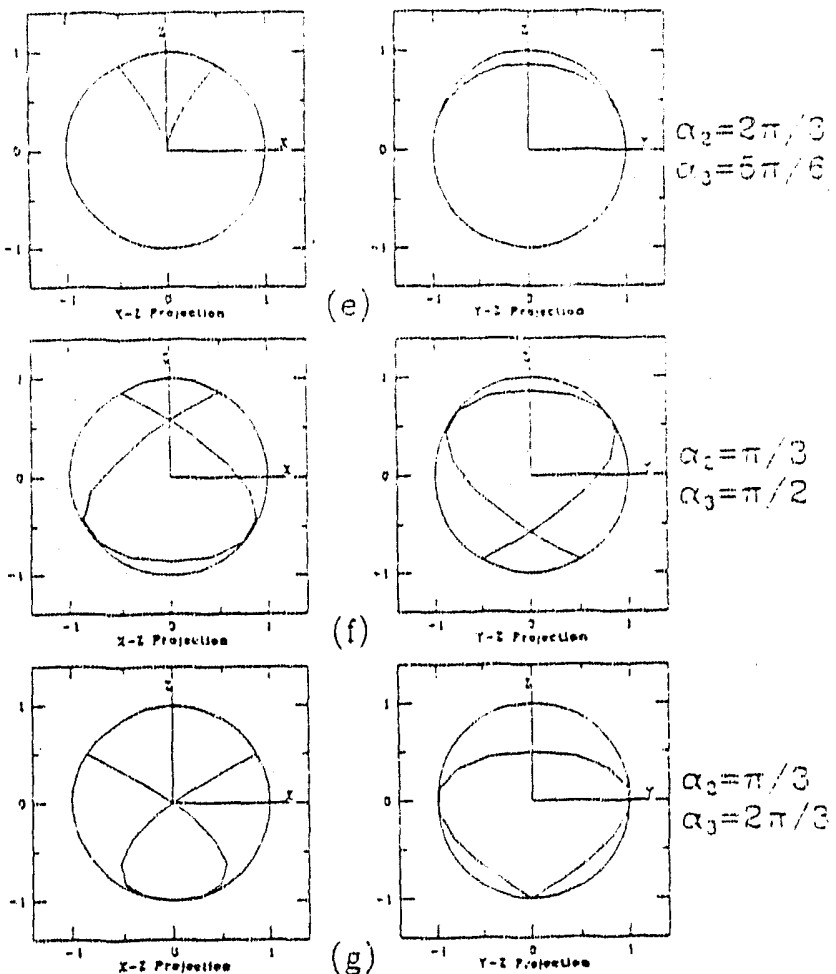

(f)
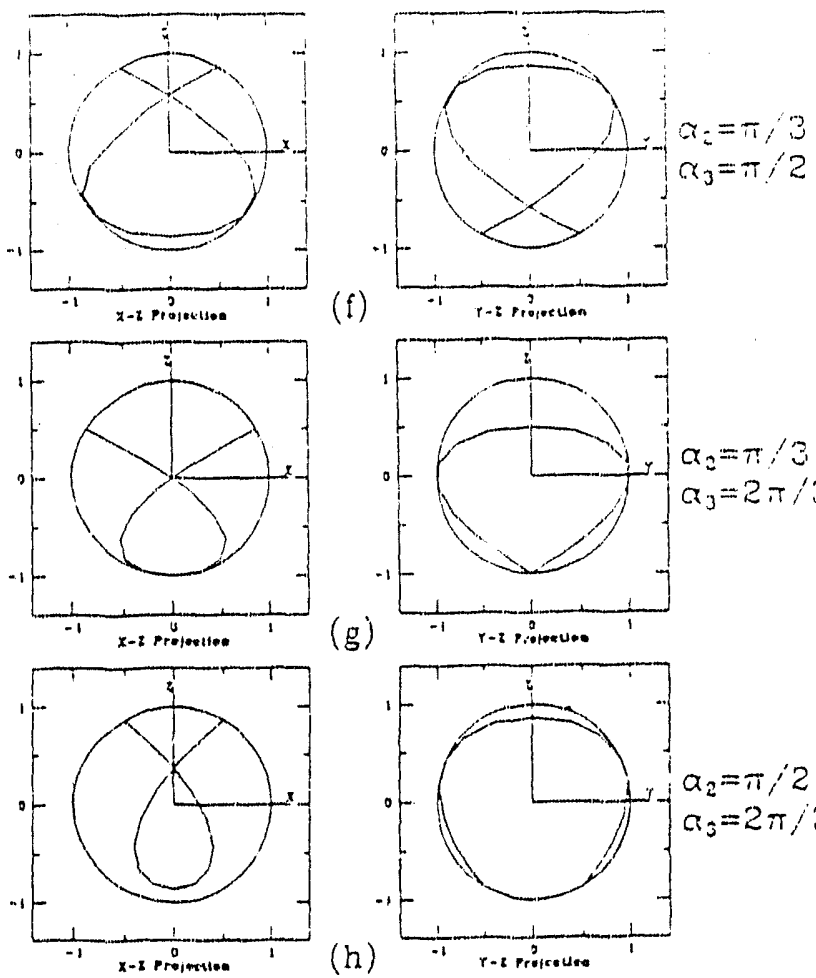

(g)

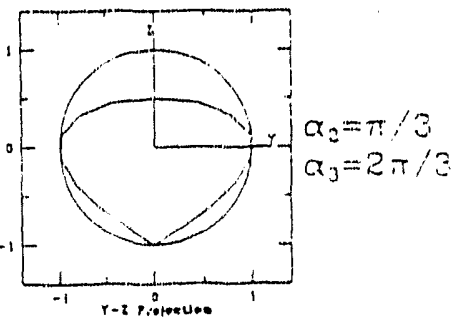

h)

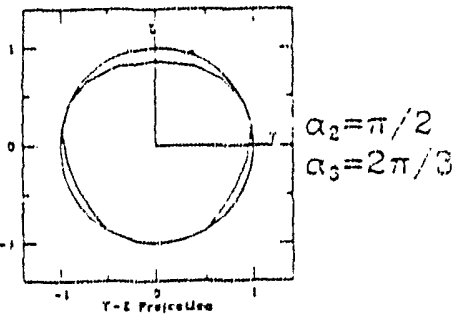

Figure ti: Typical spherical limacons, $\mathrm{N}=2$ (conlinued). 


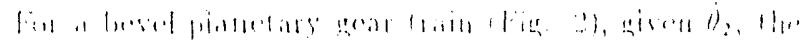

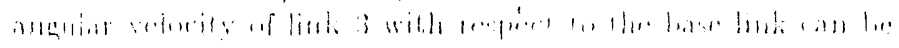

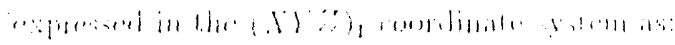

$$
\begin{aligned}
& { }^{1} \vec{w}_{3}=\dot{1}_{2} \ddot{B}_{1}+b_{1} \dot{r}_{2} \\
& =\dot{l}_{2}\left(\ddot{z}_{1}+1 \dot{m}_{2}\right) \\
& =\dot{\theta}_{2}\left[\begin{array}{c}
n \times 1)_{2} \cdots 1_{2} \\
-n(0)_{2} \cdots 2_{2} \\
11+n\left(1_{2}\right)
\end{array}\right]
\end{aligned}
$$

The magnitude of $\vec{z}_{3}$ is equal to $d, \sqrt{T+n^{2}+2 n c x_{2}}$, and its ditection is along the line of $\left(\vec{Z}_{1}+n \vec{Z}_{2}\right)$. Hence. the line passing through the origin and pointing in the direction of $\left(\vec{Z}_{1}+n \vec{Z}_{2}\right)$ is the instantaneous screw axis for the motion of link 3 with respect to link 1. As a sarics from 0 to $2 \pi$, the locus of the instantaneous screw axis forms a screw cone with its vertex located at the origin. Similary, the angular velocity vector of link 3 with rospect to link 1 can also be axpressed in the moring coordinate syetem $(X Y Z)_{2}$ as:

$$
2 \vec{\omega}_{3}=\dot{\theta}_{2}\left[\begin{array}{c}
0 \\
s \alpha_{2} \\
c \alpha_{2}+n
\end{array}\right]
$$

Entration (47,b) implies that the instantaneous screw axis is fixed in the $d \cdot Z$ plane of the carrier. Hence, it revolves around the fixed $Z_{1}$-axis as the carrien rotates about the lirst joint axis.

\section{SUMMARY}

This paper discusses the trajectories of bevel planetary gear trains. Parametric equations for the trajectory are derived using $3 \times 3$ rotation matrix. We have shown that the trajectory generated by a tracer point on the planet of a bevel pianetary gear train is analogous to that of a spur-gear train. Winen the gear ratio is equal to one, the trajectory is called spherical lima. con or spherical cardioid depending on whether the tracer point lies on the outsicle or on the pitch cone of the planet gear. When the gear ratio is equal to two and the tracer point lies on the pitch cone of the planet gear, the curve is a spherical nephroid.

The geometric properties of these trajectories are investigated, including the planes of symmetry, extent, of curves, number of nodes (cusps) and their locations. We have also shown that the criterion for existence of cusps can be interpreted geometrically as the tracer point lying on the pitch cone of the planet gear or its image cone. F!rm geometric point of view: the spherical limacon can be interpreted as the intersection of a parabolic cylinder and a unit sphere; and the spherical nephroid ican be interpreted as the intersection of Tschirnhausen's cylinder and a unit sphere.

This study is useful for a better understanding on the working space and singular points of gear-coupled robotic mechanisms. The results has been applied to the stuciy of orientational workspace of three-DOF, four-jointed splierical wrist

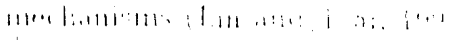

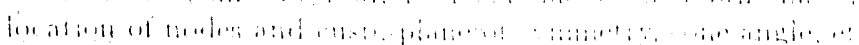

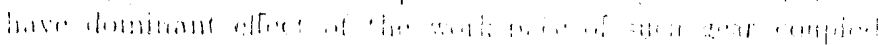

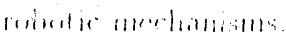

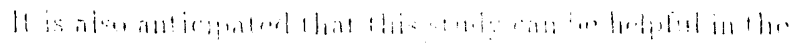

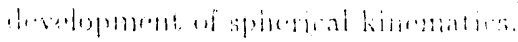

\section{Arovowhenearand}

This work was suppoted in part by the U.S. Deportment of Energy under Grant DEPO5.58ER1397\% and in part by the NSF Enginering Reseatrin Centers Pogram, NSFD CDR \$803012. Such support does not constitute an endorsement by the supporting agencies of the views expressed in the poper.

\section{REFERENCE}

1. Berser, M., and Gustianx, B., 10se, Diforntial Geom. etry: Manifolds, Curves, and Suriaces. Snringer-Verlas, New lork.

2. Briestorn, B., and Kinöner. H, 1086, Plane Algebraic Curves, Birkhäuser Boston Inc.

3. Butchart, J. H., 1945, "Some Properties of the Linncon and Cardioid," Amer. Math. Monthly, Vol. 52, pp. 3S4$3 S i$.

4. Denavit, J., and Hartenberg, R.S., 1955, "A Kinemntic Notation for Lover Pair Mechanisms Based on Malrices," ASME Journal of Applied Mechanics, Vol. 77. pp. 215. 221.

5. Hunt. K.H., 1978, Kinematic Geometry of Mechanisms, University Press, Oxford, Great Britain.

6. Jensen, P.W., 1965, "Cycloidal Cear Ifechanisms," Product Engineering, Vol. 36, No. 13, p1?. S2.85.

7. Lawrence, J. D., 1972, A Catalog of Special Plane Curves, Dover Publications, New York.

8. Lin, C.C., and Tsai, L.W., 1991, "The Wrorkspace of Three-Degree-of-Freedom, Foutr-Jointed Spherical Wrist Mechanisms," accepted for publication in the Proc. of 1091 IEEE International Conference on Robotics and Aucomation, Sacramento, CA.

9. McCarthy, J.P., 1945, "The Limncon and The Cardio:d," Math. Gazette, Vol. 29, pp. 219-220.

10. Pollitt, E.P., 1960, "Some Applications of the Cycloid in Machine Design," Trans. ASME, J. of Eng. for Industry, Series B, Vol. 82, No. 3, pp. 407.414.

11. Spragg, D., and Tesar, D., 1971, "Generalized Cycloidal Motion," Trans. ASME, J. of Eng. for Industry, Series B, Vol. 93, No. 1, pp. 131-139. 

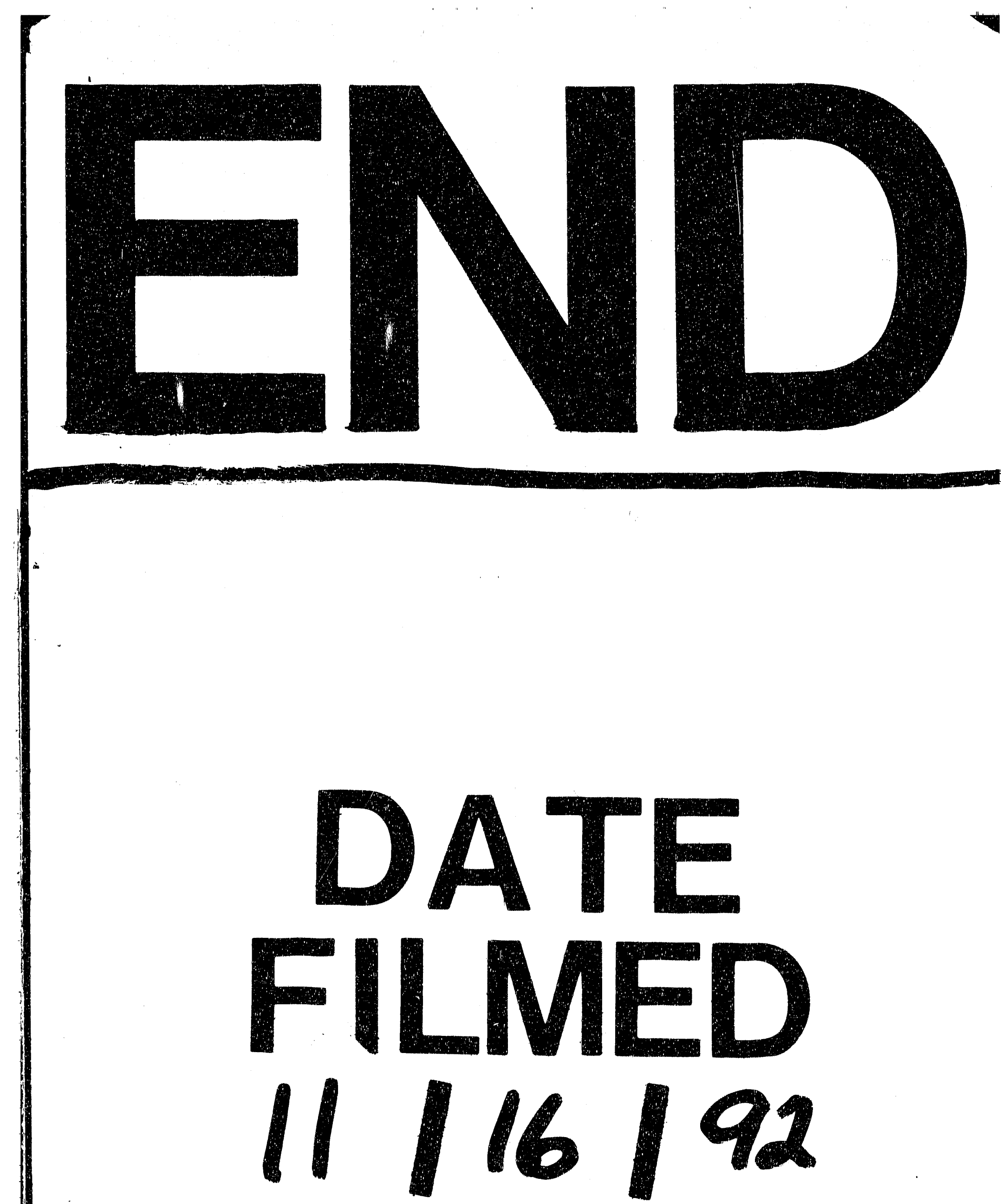


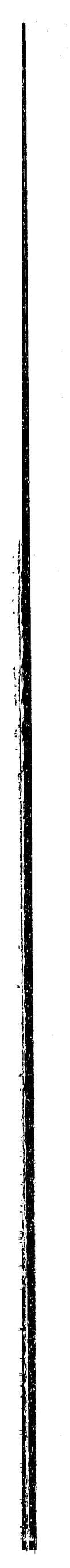

